\title{
Activity cycles of blazars and quasars from VLBI observations
}

\author{
N. A. Kudryavtseva*, ${ }^{1,2}$, S. Britzen ${ }^{1}$, M. F. Aller ${ }^{3}$, H. D. Aller ${ }^{3}$, D. C. Gabuzda ${ }^{2}$, \\ S. G. Jorstad ${ }^{4}$, H. Teräsranta ${ }^{5}$, A. Witzel ${ }^{1}$, J. A. Zensus ${ }^{1}$ \\ ${ }^{1}$ Max-Planck-Institut für Radioastronomie, Bonn, Germany \\ ${ }^{2}$ University College Cork, Cork, Ireland \\ ${ }^{3}$ Astronomy Department, University of Michigan, USA \\ ${ }^{4}$ Institute for Astrophysical Research, Boston University, Boston, USA \\ ${ }^{5}$ Metsähovi Radio Observatory, Helsinki University of Technology, Finland \\ E-mail: nadia@physics.ucc.ie
}

We discuss a new approach for measuring flaring activity cycles of blazars and quasars. The activity cycles of 21 active galactic nuclei were estimated using the radio total flux-densities at five frequencies $(5 \mathrm{GHz}-37 \mathrm{GHz})$ and high resolution long-term very long-baseline interferometry (VLBI) observations $(1.6 \mathrm{GHz}-43 \mathrm{GHz})$. We define the activity cycles as the time period between two successive "core" outbursts in the total flux-density radio light curves. The "core" outbursts show frequency-dependent time-delays, flat amplitude spectra and are associated with brightening of the VLBI core. We show that the activity cycles are very long and have durations from 4 to $>25$ years in observer's frame. We also show that a few sources, such as S5 1803+784, reveal less active behavior if we take into account the long-term VLBI and total flux-density observations for more than 20 years. We found for four sources that core flares appear periodically and that activity cycles coincide with a period in the total flux-density variability. Analysis of the activity cycles leads us to suggest that more massive blazars and quasars possess shorter activity cycles. Quasars and blazars with mass as of about $10^{8} M_{\text {sun }}$ have activity cycles of $\sim 12 \mathrm{yrs}$, whereas sources with mass as of $10^{10} M_{\text {sun }}$ have activity cycles of about 2 yrs in the source frame.

The 9th European VLBI Network Symposium on The role of VLBI in the Golden Age for Radio Astronomy and EVN Users Meeting

September 23-26, 2008

Bologna, Italy

\footnotetext{
* Speaker.

${ }^{\dagger}$ Member of the International Max Planck Research School (IMPRS) for Astronomy and Astrophysics
} 


\section{Introduction}

Total flux-density radio light curves of blazars and quasars show timescales from days up to decades of years. Various authors calculated variability timescales using various mathematical methods, such as structure functions (e.g. [5]), Fourier transforms (e.g. [8]) and wavelet transforms (e.g. [3]). However, these methods can show only the characteristic time scale of the total fluxdensity variability and do not take into account physical properties of the active galactic nuclei (AGN), such as spectral evolution and opacity of the flares.

In this proceeding, the new physical approach to calculating the activity cycles of blazars and quasars presented in [15] is discussed and developed. We can investigate the flaring activity cycle of blazars and quasars, calculating the duration of a cycle as the time between two optically thick "core" outbursts. The "core" outbursts are flares connected with a primary perturbation in the base of the jet, which show frequency-dependent time delays and are associated with brightening of the VLBI core $[4,14]$. Thus, studies of flaring activity cycles will provide information on how often a primary perturbation at the base of the jet appears and how stable the flaring activity cycles are, which will improve our understanding of the physical processes in the vicinity of the central supermassive black holes. The first results of this new physical approach were presented in $[15,16]$, and here we discuss new results for 21 sources.

\section{Observations}

The University of Michigan Radio Astronomy Observatory [1] monitoring program and Metsähovi Radio Astronomy Observatory monitoring [18] database, together with archival historical radio data, were used to investigate activity cycles in blazars and quasars. Data at 4.8, 8, 14.5, 22 and $37 \mathrm{GHz}$ spanning more than 30 years were analyzed. The total flux-density variability data were also complemented with VLBI data at $43 \mathrm{GHz}$ [6] and archival observations at six frequencies 1.6-22 GHz ( [11] and ref. therein).

For studying the properties of total flux-density outbursts we separated the most prominent flares into individual components using Gaussian model fitting. We chose to fit Gaussian components since most of the observed centimeter-wavelength outbursts are symmetrical and have shapes that are approximately Gaussian.

\section{Activity cycles}

We have selected 21 sources with bright, well-separated outbursts and definite frequencydependent time delays suitable for the analysis. The activity cycles of the sources were calculated as the time interval between two core outbursts, following the procedure discussed in $[15,16]$. We present first our results for the blazar S5 $1803+784$, which is the source with the largest amount of analyzed VLBI data.

The analysis of 20 years of structural variability in the parsec-scale jet of S5 1803+784 has shown that the jet structure within 12 mas of the core consists of seven quasi-stationary jet components, which stayed at about the same positions for the entire 20 years. Only one jet component ejection was detected at lower frequencies, in $1999.8 \pm 1.1$ (See [11, 12, 2] for more details), 

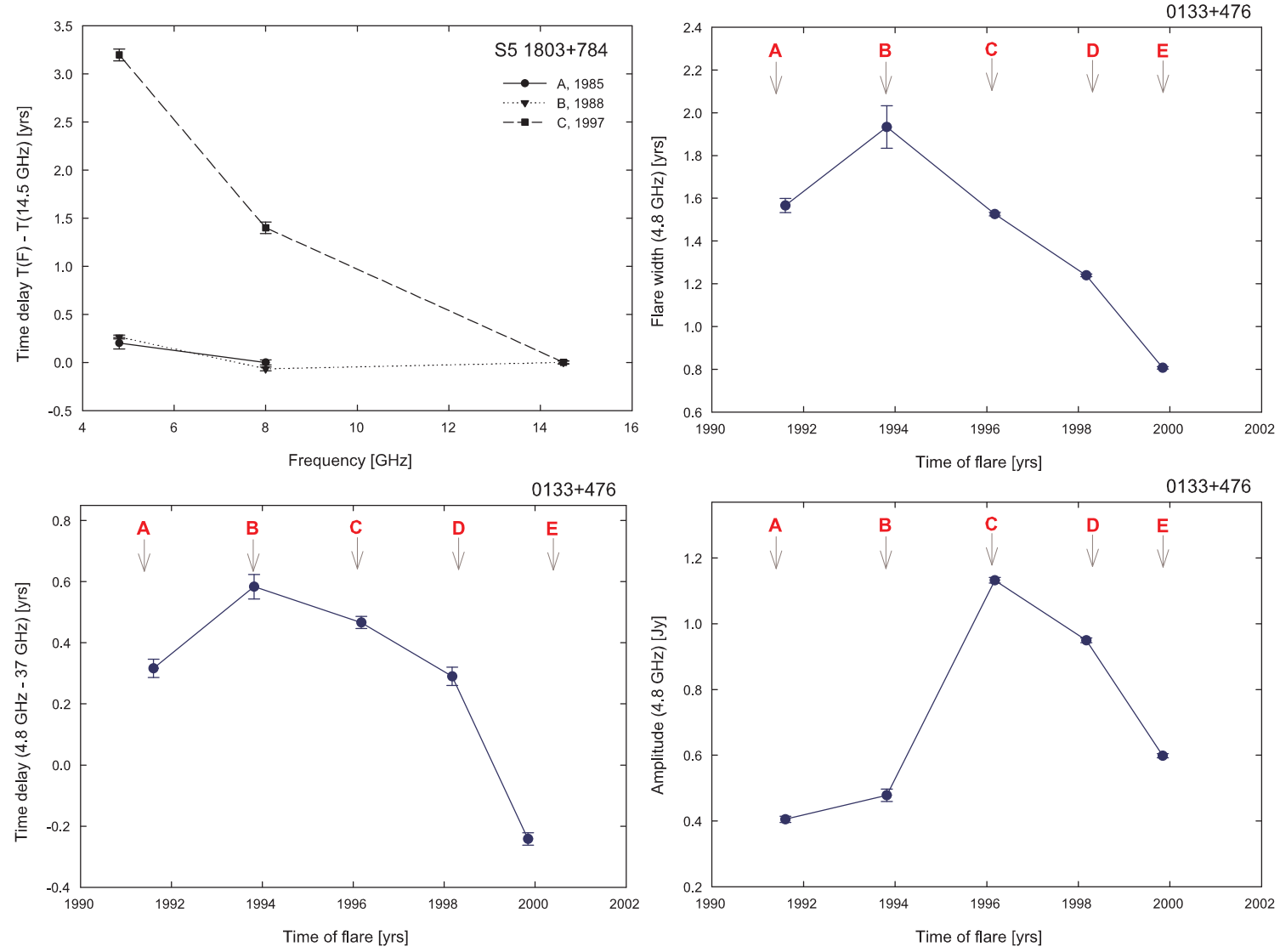

Figure 1: Top Left: Time lags of S5 1803+784 vs. frequency. Bottom Left: Time evolution of frequency-dependent time lags between $4.8 \mathrm{GHz}$ and $37 \mathrm{GHz}$, amplitudes at $4.8 \mathrm{GHz}$ (Bottom Right), and flare widths at $4.8 \mathrm{GHz}$ (Top Right) in $0133+476$. Arrows mark position of each total flux-density outburst.

which was observed as two jet components ejected in about 1998 at $43 \mathrm{GHz}$ [7]. We calculated the frequency-dependent time delays for each flare as the time difference between the Gaussian peaks at each frequency with respect to the position of the peak at the highest available frequency. Time delays for the outbursts as functions of frequency are shown in Fig. 1 (Top left). Decomposition of the total flux-density light curves shows that the light curve of S5 1803+784 consists of three flares. The first two flares show moderate time delays of 0.3 years, whereas the prolonged flare $\mathrm{C}$ (1997) shows an enormous time delay of $3.2 \mathrm{yrs}(4.8-14.5 \mathrm{GHz})$, which is an indication of high opacity in the source during this outburst. The bright flare $\mathrm{C}$ is accompanied by the significant flattening of the spectra, high opacity, and brightening of the core, and is followed by ejection of a jet component. Therefore, we classified C flare as a "core" flare and since it was the only "core" flare observed for more than 25 years of observations the activity cycle of S5 1803+784 is more than 25 years.

The total flux-density light curves and VLBI structure variability were analyzed in a similar way for the other sources. Table 1 shows the list of analyzed active galactic nuclei (15 quasars, 2 blazars and 1 radio galaxy) and gives the source name, class, redshift, activity-cycle duration in the observer's frame, activity-cycle duration in the source frame, mass and reference for the mass estimation. The cycles tend to be long, about 4-6 years in the source frame (distribution maximum 

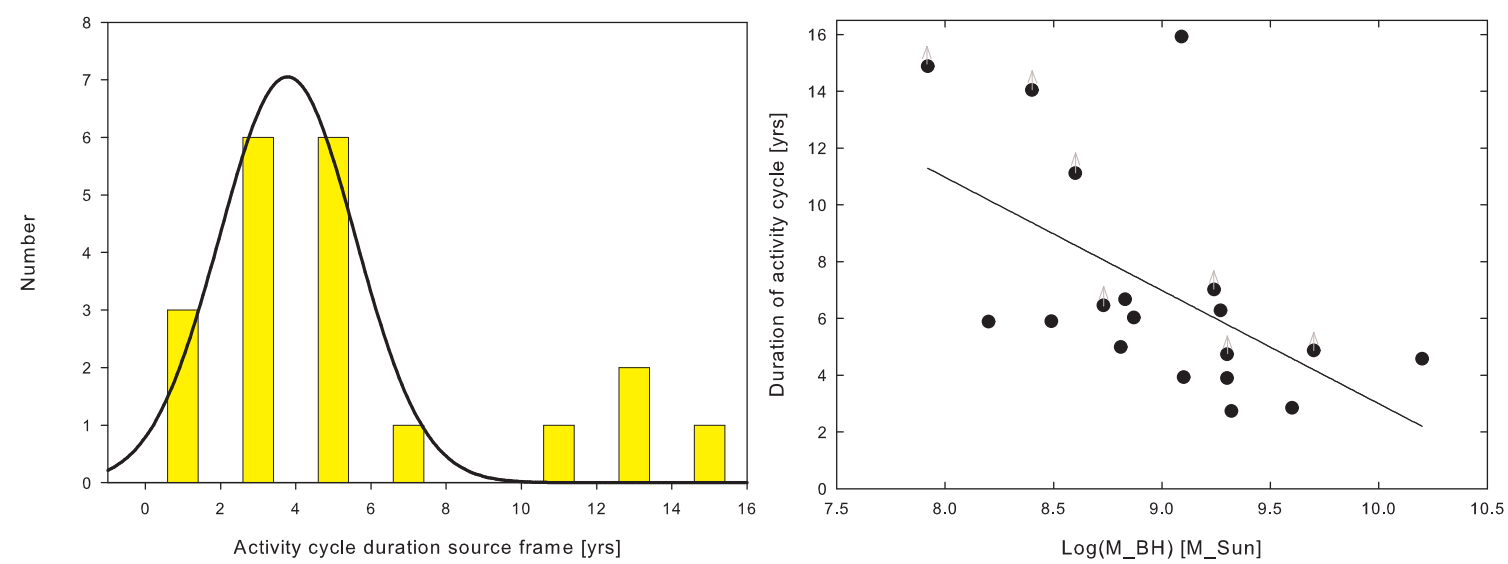

Figure 2: Left: Number of sources with particular duration of activity cycle. The length of each activity cycle is shown in the source frame; Right: Correlation between duration of activity cycles in the source frame and mass of supermassive black hole in the center of a galaxy.

at $3.8 \pm 0.3 \mathrm{yrs})$. This corresponds to a cycle duration of about $8-12$ years in the observer's frame for $z=1$. Figure 2 (Left) shows the distribution of the activity-cycle durations in the source frame.

The duration of the activity cycle correlates with the logarithm of the mass of the supermassive black hole at the center of a galaxy, with the correlation coefficient $r=-0.6$. Moreover, if we exclude sources with upper limits for the activity cycles, the correlation coefficient becomes higher, $r=-0.8$. Figure 2 (Right) shows this relation between the cycle duration in the source frame and the mass. This plot suggests that, on average, blazars and quasars with lower-mass black holes have longer flaring activity cycles. We have also searched for correlations between the activity-cycle duration and the apparent speeds of the jet components, quiescent flux at $14.5 \mathrm{GHz}$, maximum amplitude, time delay and width of the fitted Gaussian functions. We found a tentative correlation between the activity-cycle duration and maximum flare width at $37 \mathrm{GHz}$, which suggests a possible correlation between the activity-cycle duration and the duration of flares. However, we did not find significant correlations between the activity cycles and other source properties, such as apparent speeds and time delays and amplitudes of the flares.

Time evolution of activity cycles: For six sources (0133+476, 0735+178, 3C 345, 2145+067, $3 \mathrm{C} 446$, and $3 \mathrm{C} 454.3$ ) we found that the presence of the flaring activity cycle is demonstrated not only by repeatability of the core flares, but also by smooth and gradual changes of the frequencydependent time lags, flare durations, amplitudes and spectra. These properties of the flares change gradually from flare to flare during the activity cycle. This is shown in Fig. 1 for the quasar 0133+476, where the time lags, amplitudes and widths for all observed flares (A-E) are plotted versus time. Moreover, the time evolution of the amplitude, frequency-dependent time delays and durations of the flares are correlated for all six sources. In a few cases, jet component ejections were detected when the frequency-dependent time lags and opacity reached the maxima and the emission started to become optically thinner, in agreement with the shock in jet model.

Blazars and quasars with periodic core flares: For four sources (3C 345, 3C 446, CTA 102, and $3 \mathrm{C} 454.3$ ) we found that core flares appear periodically, and that the activity cycles coincide with a period in the total flux-density variability. The "core" outbursts are probably connected with the immediate vicinity of a supermassive black hole in the center of the galaxy, and are caused 
Table 1: List of analyzed sources

\begin{tabular}{llllllll}
\hline Source & Name & Class & $\mathrm{z}$ & $\begin{array}{l}T_{a c t} \\
{[\mathrm{yrs}]}\end{array}$ & $\begin{array}{l}T_{a c t, s f} \\
{[\mathrm{yrs}]}\end{array}$ & $\begin{array}{l}\log \left(M_{B H}\right) \\
{\left[M_{\text {Sun }}\right]}\end{array}$ & $\begin{array}{l}\text { Ref. } \\
\text { mass }\end{array}$ \\
\hline $0059+581$ & & QSO & 0.644 & 4.5 & 2.7 & - & - \\
$0133+476$ & DA 055 & QSO & 0.859 & $>12$ & $>6.5$ & 8.73 & {$[21]$} \\
$0202+149$ & 4 C+15.05 & QSO & 0.405 & 4.0 & 2.8 & 9.60 & {$[13]$} \\
$0316+413$ & 3C 84 & RG & 0.017 & 6.0 & 5.9 & 8.49 & {$[21]$} \\
$0458-020$ & & QSO & 2.286 & $>16$ & $>4.9$ & 9.70 & {$[13]$} \\
$0528+134$ & & QSO & 2.060 & 14.0 & 4.6 & 10.20 & {$[13]$} \\
$0735+178$ & OI 158 & BLL & 0.424 & $>20$ & $>14.1$ & 8.40 & {$[13]$} \\
$0923+392$ & 4C 39.25 & QSO & 0.695 & $>27$ & $>15.9$ & 9.09 & {$[22]$} \\
$0945+408$ & 4C 40.25 & QSO & 1.249 & $>25$ & $>11.1$ & 8.60 & {$[22]$} \\
$1308+326$ & & QSO & 0.996 & $>14$ & $>7.0$ & 9.24 & {$[20]$} \\
$1510-089$ & & QSO & 0.36 & 8.0 & 5.9 & 8.20 & {$[22]$} \\
$1641+399$ & 3C 345 & QSO & 0.593 & 10.0 & 6.3 & 9.27 & {$[22]$} \\
$1730-130$ & NRAO 530 & QSO & 0.902 & $>9$ & $>4.7$ & 9.30 & {$[13]$} \\
$1739+522$ & 4C 51.37 & QSO & 1.375 & 6.5 & 2.7 & 9.32 & {$[22]$} \\
$1741-038$ & & QSO & 1.054 & 8.0 & 3.9 & 9.30 & {$[13]$} \\
$1803+784$ & & BLL & 0.680 & $>25$ & $>14.9$ & 7.92 & {$[22]$} \\
$2145+067$ & 4C 06.69 & QSO & 0.990 & 12.0 & 6.0 & 8.87 & {$[22]$} \\
$2223-052$ & 3C 446 & QSO & 1.404 & 12.0 & 5.0 & 8.81 & {$[20]$} \\
$2230+114$ & CTA 102 & QSO & 1.037 & 8.0 & 3.9 & 9.10 & {$[13]$} \\
$2251+158$ & 3C 454.3 & QSO & 0.859 & 12.4 & 6.7 & 8.83 & {$[22]$} \\
\hline
\end{tabular}

by the appearance of a primary perturbation at the base of the jet. Thus, periodicity in the "core" outbursts can be interpreted as evidence for periodic processes in the immediate vicinity of the black hole.

For example, in 3C 345, the activity cycle of about 10 years coincides with the period of 10.1 years found for the optical light curve [23] and the period of $\sim 9$ years found by [9] for the jet structure evolution. Moreover, the periodicity in the radio variability for 3C 454.3 and 3C 446 also coincides with the period in the optical light curve (see [10] for more details). Such coincidences of optical and radio variability were observed before in only a few sources. According to [17], such coincidences are most likely due to the presence of a binary black hole in the galactic nucleus. Given also our detection of periodicity in the "core" flares, opacities and spectra of these sources, they become the best known candidates for supermassive binary black holes, together with OJ 287 [19].

\section{Discussion}

We have applied the new method of [15] for calculating flaring activity cycles, where the flaring activity cycle is defined as the characteristic time scale between "core" outbursts. "Core" outbursts are optically thick flares with large frequency-dependent time delays and special spectral evolution, and are connected with the appearance of a primary perturbation in the jet. We have 
successfully identified "core" and "jet" outbursts for 21 sources, based on the shock-in-jet theory predictions [4] and our derived flaring activity cycles. The flaring activity cycles can last up to tenths of years. During the flaring activity cycle for six sources $(0133+476,0735+178,3 \mathrm{C} 345$, 2145+067, 3C 446, and 3C 454.3), the frequency-dependent time delays, opacity and spectral evolution change gradually with time, and are connected with jet-component ejection when the opacity reaches its maximum (in accordance with the shock-in-jet model). Thus, we are actually observing the substructure of powerful outbursts rather than single outbursts.

Based on our analysis of the long-term multi-frequency light curves and VLBI observations, we conclude that the flaring activity cycles in these sources last from 4 years up to $>25$ years in the observer's frame, and that most of the sources have activity cycles of about 4-6 years in the source frame. This means that the appearance of a primary perturbation at the base of the jet, which causes brightening of the VLBI core, powerful total flux-density outbursts and the emergence of new jet component(s), is a rare event, and long-term observations are necessary in order to study the appearance of these perturbations. Moreover, we have found that the duration of the activity cycles in the source frame and the mass of the central black hole are correlated, with AGN with lower-mass black holes, on average, having longer flaring activity cycles.

\section{Acknowledgments}

N.A. Kudryavtseva was supported for this research through a stipend from the International Max Planck Research School (IMPRS) for Astronomy and Astrophysics. This publication has emanated from research conducted with the financial support of Science Foundation Ireland. This research has made use of data from UMRAO which is supported by the NSF and by funds from the University of Michigan. We acknowledge the MOJAVE and VLBA 2-cm Survey Program teams.

\section{References}

[1] H. D. Aller, M. F. Aller, G. E. Latimer, P. E. Hodge, 1985, ApJS, 59, 513

[2] S. Britzen, N. A. Kudryavtseva, A. Witzel et al., accepted for publication to A\&A

[3] A. Ciaramella, C. Bongardo, H. D. Aller et al., 2004, A\&A, 419, 485

[4] J. L. Gómez, J. M. A. Martí, A. P. Marscher, J. M. A. Ibáñez, \& A. Alberdi, 1997, ApJ, 482, 33

[5] P. A. Hughes, H. D. Aller, M. F. Aller, 1992, ApJ, 396, 469

[6] S. G. Jorstad, A. P. Marscher, J. R. Mattox et al., 2001, ApJS, 134, 181

[7] S. G. Jorstad, A. P. Marscher, M. L. Lister et al., 2005, AJ, 130, 1418

[8] B. C. Kelly, P. A. Hughes, H. D. Aller, M. F. Aller, 2003, ApJ, 591, 695

[9] J. Klare, J. A. Zensus, A. P. Lobanov et al., ASP Conf. Ser., 2005, 340, 40

[10] N. A. Kudryavtseva, T. B. Pyatunina, 2006, ARep, 50, 1

[11] N. A. Kudryavtseva, S. Britzen, A. Witzel et al., 2006a, the 8th EVN Symposium, 7, PoS 8thEVN 007

[12] N. A. Kudryavtseva, PhD dissertation, University of Cologne, 2008

[13] E. W. Liang, H. T. Liu, 2003, MNRAS, 340, 632

[14] A. P. Marscher, 1996, ASP Conf. Ser. 110, 248 
[15] T. B. Pyatunina, N. A. Kudryavtseva, D. C. Gabuzda et al., 2006, MNRAS, 373, 1470

[16] T. B. Pyatunina, N. A. Kudryavtseva, D. C. Gabuzda et al., 2007, MNRAS, 381, 797

[17] F. M. Rieger, 2005, ChJAS, 5, 305

[18] H. Teräsranta, M. Tornikoski, A. Mujunen et al., 1998, A\&AS, 132, 305

[19] Valtonen, M. J., 2007, ApJ, 659, 1074

[20] J.-M. Wang, B. Luo, L. C. Ho, 2004, ApJ, 615, 9

[21] J.-H. Woo, C. M. Urry, 2002, ApJ, 579, 530

[22] Y. Liu, D. R. Jiang, M. F. Gu, 2006, ApJ, 637, 669

[23] X. Zhang, G. Z. Xie, J. M. Bai, 1998, A\&A, 330, 469 